\title{
Development and Evaluation of a Pictogram for Thai Patients with Low Literate Skills
}

\author{
W. PHIMARN*, L. RITTHIYA, R. RUNGSOONGNOEN, W. PATTARADULPITHUK AND K. SARAMUNEE \\ Social Pharmacy Research Unit, Faculty of Pharmacy, Mahasarakham University, Kantharawichai, Maha Sarakham 44150, \\ Thailand
}

Phimarn et al.: Evaluation Pictogram for Thai Low Literate Skills

\begin{abstract}
The patients with low literacy have difficulty in understanding instruction in medication labels. International pictograms would help resolve this problem. The purpose of the study is to develop a local pictogram for low literate Thai patients and to evaluate its effectiveness in improving patient's understanding of prescription directions, medication adherence, and satisfaction. A four phase, mixed method study was designed. In phase I, a brainstorming discussion $(n=21)$ was used to gather essential information to devise the first draft pictogram. An interview was used in phase II $(n=20)$ and III $(n=30)$ to adjust and finalize the pictogram. In phase IV, a randomized controlled trial was conducted. Low-literate and poor adherence patients were recruited from primary healthcare in northeast Thailand. The experimental $(n=67)$ and control $(n=67)$ groups received medications with attached final pictogram and traditional labels, respectively. Participants were assessed understanding and adherence of medication use on day 0 and 14 with a reminder on day 7. Satisfaction of pictogram use was also evaluated on day 14. The experimental group had post intervention understanding scores that were significantly higher than the control group $(100.00$ vs. $97.10 \pm 3.64 ; p<0.046)$. Moreover, the adherence score in the experimental group was statistically higher than the control group $(97.41 \pm 3.62$ vs. $95.84 \pm 4.72 ; p<0.033)$. Most participants in the experimental group were satisfied with the final pictogram and also agreed that the local pictogram may improve their understanding and adherence. Pictograms improved both understanding and adherence among low literate participants.
\end{abstract}

Key words: Literacy, medication information, pictograms, Thai patients

Low literacy is an important and widespread problem. Deficiencies in basic reading, communicating, and comprehension skills significantly affect the ability to function in daily life, particularly effective use of medicines, as patients cannot perform necessary functions in health care environment such as understanding the directions on the label on medication packages $^{[1]}$. In 1990 , the UNESCO reported $26.6 \%$ of adults in the world were illiterate and of those $34.9 \%$ were in developing countries ${ }^{[2]}$. Illiteracy rate was found quite high (30\%) in Asia, whereas it was as low as $1 \%$ in developed countries ${ }^{[3]}$. In Thailand, the report of literacy survey illustrated that Thai people were approximately $95 \%$ literate $^{[4]}$. The Thai National Statistical Institute reported more than $7.5 \%$ of Thai people over the age of 50 were illiterate ${ }^{[5]}$.

Level of literacy has an impact on health literacy, which means the cognitive and social skills of individuals to gain access to understand and use promoted health information for maintaining good health ${ }^{[6]}$. Inadequate health literacy may therefore decrease abilities of people to understand health information. In addition, people with low literacy are likely to have difficulties in understanding language/instruction in medication labels ${ }^{[6,7]}$. This problem results in poor health outcomes and high healthcare costs ${ }^{[8-10]}$. Previous studies reported that abilities to read ${ }^{[11,12]}$ and understand medication ${ }^{[13]}$ labels are important to medication adherence. One way to facilitate communication of medication information with patients, who literacy skills are limited, is to incorporate visual aids; such as graphic symbols or pictograms, on medication labels and leaflets ${ }^{[14]}$.

This is an open access article distributed under the terms of the Creative Commons Attribution-NonCommercial-ShareAlike 3.0 License, which allows others to remix, tweak, and build upon the work non-commercially, as long as the author is credited and the new creations are licensed under the identical terms

Accepted 25 November 2018

Revised 24 April 2018

Received 05 May 2017

Indian J Pharm Sci 2019;81(1):89-98 
Pictograms can communicate or translate meanings without a written language. They can be recognized and transmitted to a message more easily, naturally and effectively, compared to using words. The United States Pharmacopoeia (USP) is the leading international source, which provides a set of standard pharmaceutical pictograms ${ }^{[15]}$. In spite of its effectiveness, some limitations are noted by the International Pharmaceutical Federation. Visual images may need to adjust and validate in accordance with culture, beliefs and attitudes of different countries or continents ${ }^{[16,17]}$. Previous research indicated that the environment, both ecological and cultural, could exert a significant influence on the ability of an individual to perceive a message from pictures ${ }^{[18-20]}$. This also happens in Thailand, where various cultures appear in one country, not all symbols of the USP-DI pictograms are easy to understand for Thai people. Therefore, there is a need to develop pictograms that are based on culture of each part of the country.

This study was designed to utilize culturally adapted pharmaceutical pictograms for patients in northeastern Thailand who have low literacy and to evaluate their effectiveness in incremental understanding of prescription directions, medicine adherence, and satisfaction. Furthermore, a comparison was made between the use of the final pictograms and the traditional labels.

\section{MATERIALS AND METHODS}

A mixed-method study was designed and divided into four phases using various methods: brainstorming, interviews, pilot evaluation to finalize pictogram and randomized controlled trial (RCT). This study was approved by the research ethics committee, Mahasarakham University (Ref: 122/2555). Research information was given to all participants before the consent was obtained.

\section{Phase I, brainstorming to develop the first draft pictogram, literacy test:}

The medication use literacy test (MULT) for Thai people, developed by Chaijinda ${ }^{[21]}$, was used. The test comprised of 10 items on a 2-point (yes/no) response scale. The total score was calculated by the sum score of the test. The MULT score that is equal or less than eight indicates the low literacy in medication use; above eight denotes a literate person. The reliability of this test was satisfactory with Chronbach's alpha of 0.845 .

\section{Recruitment of participants:}

A brainstorming discussion was conducted to obtain essential information of which pictogram should be featured. This discussion was for the purpose of gathering opinions from the various stakeholders, low literacy (MULT $\leq 8$ ), pharmacy students representing literate persons (MULT $>8$ ), academic and hospital pharmacists. Healthcare professionals were included since their expertise in health and medicines was of value to design the appropriate pictograms. An invitation to participate in this discussion was publicized at various locations that include, nearby villages, pharmacy school and hospitals. Members of the general public and pharmacy students who expressed an interest in taking part in the study were asked to complete MULT in order to quantify their literacy level.

\section{Conduct of a brain storming discussion:}

A $3 \mathrm{~h}$ brain storming discussion was held at a temple, where a multi-purpose pavilion was provided for public use. Participants were provided with brief information of the research prior to signing a written consent form. Authors LR and RR facilitated the discussion while the others assisted in taking notes. The brain storming focused on how local pictograms representing directions of medicine use should be figured/drawn. Topics discussed were the following; picture(s)/ feature(s) that can reflect time for taking medicine, e.g. in the morning, noon, evening or at bedtime, picture(s)/ feature(s) that can reflect a meal or food, picture(s)/ feature(s) that reflect the word 'before' and 'after', picture(s)/feature(s) that can represent a tablet.

\section{The first draft pictograms:}

Based on the results from brain storming, one of the investigators (WP) initially designed the pictograms by adapting from those previously developed by USPDI. Symbols were sketched based on northeastern Thai culture. The first draft of seven pictograms representing directions of medicine use were roughly sketched by WP: four reflected different times of day (morning, noon, evening and bedtime); two represented taking medicine before and after meal; one represented a tablet. Several pictograms were drawn for each direction for the assessment in phase II.

\section{Phase II, interview to finalize the pictogram, comprehension test:}

This study used American National Standards Institute (ANSI) standard on Criteria for Safety Symbols to 
assess comprehension level of pictograms. The ANSI score is calculated in percent by number of participants correctly answering the meaning of pictogram, comparing to the intended definition, divided by the total number of participants. An $85 \%$ comprehension rate is the minimum cutoff for acceptability ${ }^{[22]}$.

\section{Recruitment of participants and conduct of interview:}

The interview was to assess the first draft pictogram, intentionally with various stakeholders: low literate, literate persons and health care professionals. Participants were recruited as in the same way as used in phase I. Participants were asked to look at a series of the first draft pictograms and their comprehension was tested ${ }^{[23,24]}$. The answers given by participants were then compared to the intended meaning to calculate the ANSI score. Participants were also asked to make further suggestions to the first draft pictograms. Three pictograms of each direction, 21 in total, with the ANSI score $\geq 85 \%$ were selected since they transmitted the message correctly to most participants. The selected pictograms were revised in accordance with participants' suggestions, making the adjusted pictograms for use in phase III.

\section{Phase III, pilot evaluation to finalize pictogram, recruitment of participants:}

Pilot evaluation of pictogram was conducted among general public to finalize the pictogram. An invitation to participate in this phase was publicized through community radio center. Participants were asked to complete the literacy test prior to taking part to ensure a good mix of literacy level of participants.

\section{Pilot evaluation:}

Participants were asked to interpret the meaning of the adjusted pictograms (fig. 1), comparing to the intended definition. The pictograms with the highest ANSI score were then selected to use as the final pictogram. Participants were also asked to make further suggestions on the selected ones before adjusting and implementing in the RCT.

\section{Phase IV, randomized controlled trial:}

A RCT was undertaken from June 2013 to February 2014 in four provincial areas of northeastern Thailand, to compare effectiveness of the final pictogram and the traditional label. Low literacy patients (MULT score $\leq 8$ ) with poor medication adherence (adherence $<80 \%$, assessed by patient medical history) were recruited at sub-district health promoting hospitals and a centre for primary care. Optimal sample size was calculated using the formula below. Parameters used for calculation were based on previous research ${ }^{[20]}\left(Z_{\alpha}=1.96, Z_{\beta}=0.84\right.$, $\mathrm{S}^{2}\left(\mathrm{SD}_{1}+\mathrm{SD}_{2}\right)=7.40+0, \mathrm{~d}^{2}(95.86-100)^{2}$. This suggested the appropriate number of participants should be at least 51 in each group. $\mathrm{n}=(1.96+0.84)^{2} \times 2 \times\left(7.4^{2}+0^{2}\right) /$ $(95.86-100)^{2}$.

Inclusion criteria were as follows: $18 \mathrm{y}$ of age or older; MULT score $\leq 8$; being prescribed medications consisting of at least one solid oral dosage form for chronic disease; poor medication adherence; taking medicines by themselves without the help of caregiver; never been educated about pictograms from elsewhere.

\section{Intervention:}

A flowchart describing the interventions used for both groups is shown as fig. 2. Patients in the control group received medications with attached traditional labels such as directions of medicine use described in plain text. Those in the experimental group received medications with attached final pictogram (fig. 3) that were designed as previously mentioned. Agreed participants were randomly assigned to either the control or the experimental group by draw lots technique. A box of lots was provided, then one label was drawn by a participant to assign which group he/ she would be in. Five pharmacists who had regular schedule to work at sub-district health promoting hospitals were involved in this RCT. They were trained by the research team on how to advise patients on the use of pictogram labels before implementing the study. At day 0, pharmacists counseled patients at the time of dispensing. Patients in both groups were educated about their medications based on their own labels; traditional labels or final pictogram. Patients were asked to explain back to the pharmacist about how they were going to use the prescribed medications to ensure patient's comprehension. They were reminded regarding medication use at day 7 .

Demographic data were collected including gender, age, occupation, monthly income and education level. Since, hospital pharmacists generally follow up poos adherence patient every two weeks. Thus, understanding of prescription directions and adherence were assessed at day 0 and 14 . Satisfaction of the final pictogram was also evaluated at day 14 .

\section{Understanding of prescription directions:}

Patients' understanding of prescription direction was 


\section{Prescription direction}

Taking in the morning

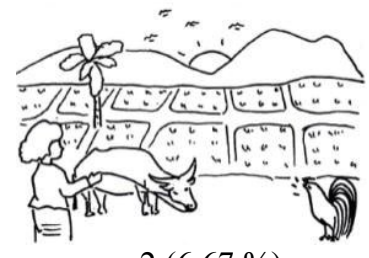

$\mathrm{n}=2(6.67 \%)$

Taking at noon

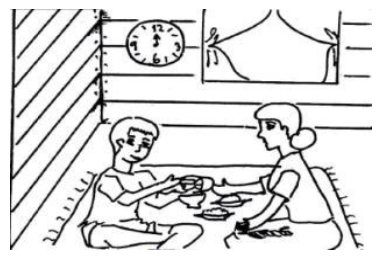

$\mathrm{n}=1(3.33 \%)$

Taking in the evening
Taking at bedtime

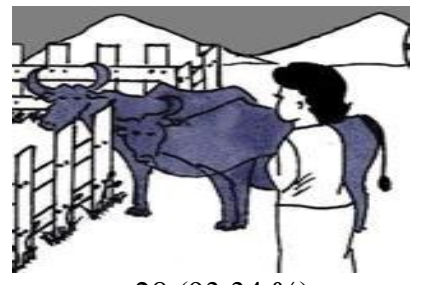

$\mathrm{n}=28(93.34 \%)$
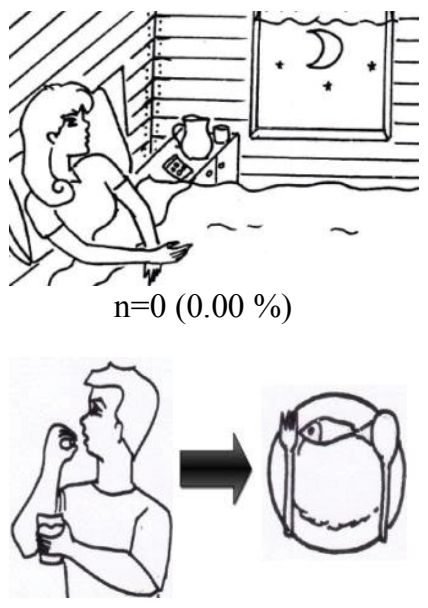

$\mathrm{n}=27(90.00 \%)$

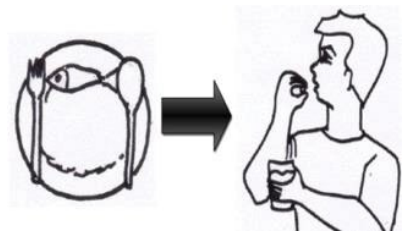

$\mathrm{n}=26(86.66 \%)$

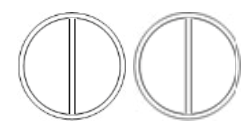

$\mathrm{n}=1(3.33 \%)$

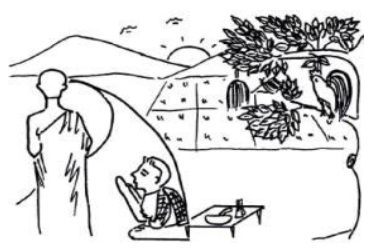

$n=26(86.66 \%)$
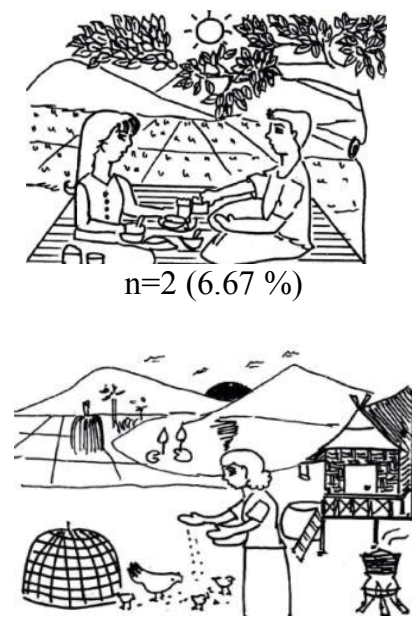

$n=1(3.33 \%)$

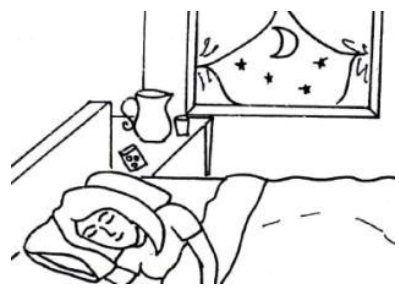

$n=2(6.67 \%)$
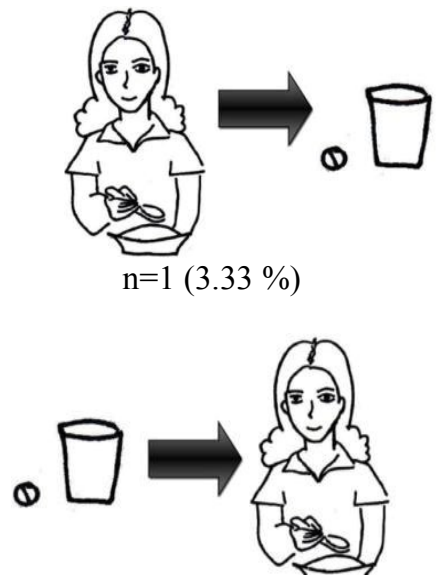

$\mathrm{n}=2(6.67 \%)$

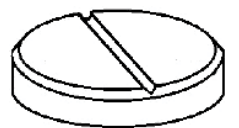

$\mathrm{n}=1(3.33 \%)$

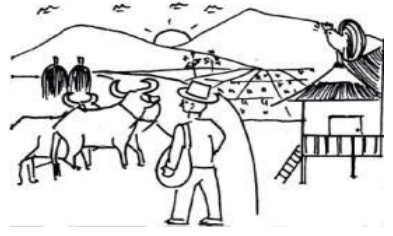

$\mathrm{n}=2(6.67 \%)$
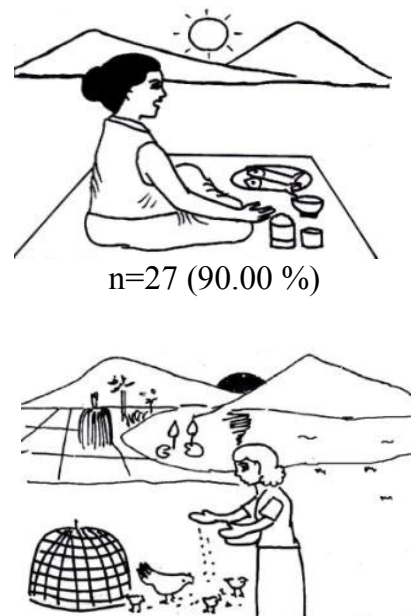

$\mathrm{n}=1(3.33 \%)$

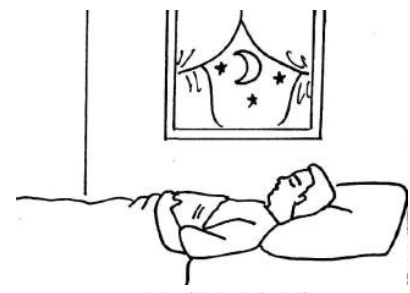

$\mathrm{n}=28(93.34 \%)$

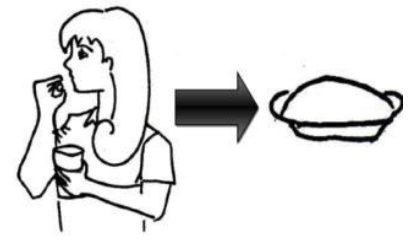

$\mathrm{n}=2(6.67 \%)$

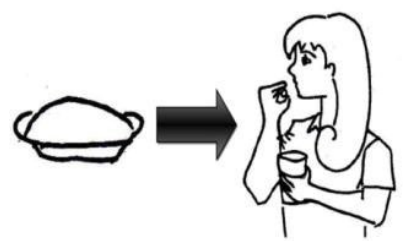

$\mathrm{n}=2(6.67 \%)$

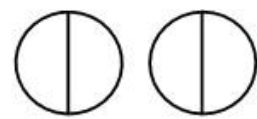

$\mathrm{n}=28(93.34 \%)$

Fig. 1: The adjusted pictograms with ANSI score

The adjusted pictogram (phase II) and number of participants understanding the pictogram correctly with ANSI score (phase III). $\mathrm{N}$ is the number of participants who understand each pictogram correctly. ANSI score is reported as percent in brackets 
developed by Chaijinda ${ }^{[21]}$. The understanding scores were assessed as per one medicine that patients used by these questions: how many tablets to be taken at a time, frequency of dose, when to take medication in relation to meals and time of day. Each correct direction explained by patient was scored as one point. Interpretation score was calculated by summing up the scores of those items and converted to percent. Average score was computed as an overall understanding score. The understanding score ranged from 0 to $100 \%$ For example, patient A was prescribed two drugs, hydrochlorothiazide (HCTZ) $25 \mathrm{mg}$ OD and metformin $500 \mathrm{mg}$ tid. If the patient explained that he/she took one tablet of HCTZ $25 \mathrm{mg}$ in the morning, then the understanding score for HCTZ is $100 \%(3 / 3)$. Then he/ she described that he/she took metformin $500 \mathrm{mg}$ twice daily, then the understanding score for metformin is $60 \%(3 / 5)$. The average understanding score is $80 \%([100+60] / 2)$.

\section{Adherence measure:}

Patients were asked the amount of doses they had taken and a dose count of remaining tablets was conducted. The degree of adherence was calculated by using the following Eqn ${ }^{[2]}, \mathrm{C}=100 \times[\mathrm{a}-|\mathrm{a}-\mathrm{b}|] / \mathrm{a}$, where, $\mathrm{C}$ is adherence, expressed as a percent; $\mathrm{a}$ is the amount of medication that should be taken if the prescriber's instructions have been followed, starting from day 0 to day 14; $b$ is the amount of remaining medication obtained from the patient minus the amount left on the follow up interview day. The degree of adherence rate ranged from 0 to 100 percent. The average mean of adherence rate was calculated if the patient took more than one medication item.

\section{Satisfaction:}

A form for assessing patient satisfaction with labeling system was developed by Chaijinda ${ }^{[21]}$. This questionnaire consisted of 14 items covering necessary contents about pictogram implementation such as size, color, position and graphics. A five-point scale was used for all items; $1=$ strongly disagree $5=$ strongly agree.

\section{Data analysis:}

Statistical analysis was performed using the statistical package for the social sciences (SPSS) version 16. Descriptive statistics were used to report results as mean and standard deviation and percentage, as appropriate. Statistical differences within the same group were tested using paired t-test while independent t-test was used for testing difference between two groups. The level of statistical significance was determined to be 0.05 or less.

\section{RESULTS AND DISCUSSION}

There were 21 participants in phase I were 10 low literate persons $(\mathrm{MULT} \leq 8), 5$ pharmacy students (MULT $>8), 3$ academics and 3 hospital pharmacists. Participants aged between 20-65 y, 13 were female. Brainstorming participants preferred these symbols to display different times of the day: the sun, the moon, stars, lightness and darkness. The sun and the moon seemed appropriate to remind low literates about time of taking medicine. A picture of sunrise was indicated as the sun rising at the horizon. Noon time was marked by the full sun shining in the sky. Sunset was marked by the sun setting at the horizon. Night time was presented by the moon and stars in the sky. An arrow, originated

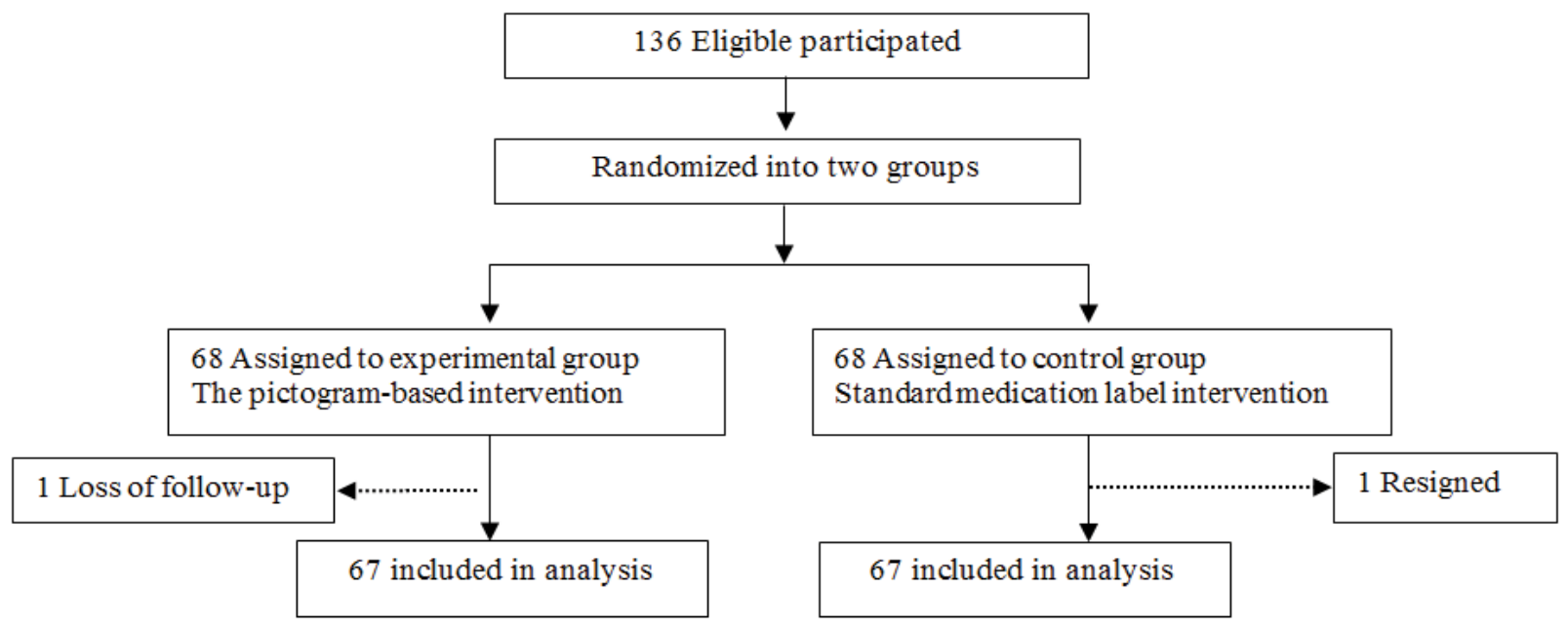

Fig. 2: Flow-chart of participants in the trial 
from left to right, was a symbolic direction to represent the word 'before' and 'after'. A meal was illustrated by rice and fish.

Twenty participants involved in phase II were 10 low-literacy, 5 pharmacy students and 5 hospital pharmacists. Participants aged between 20-60 y, 14 were female. Three pictograms of each direction that transmitted the message correctly (ANSI score $\geq 85 \%$ ) to most participants are presented in fig. 2 to be used in phase III.

Thirty general public involved in phase III were, 15 low-literate $($ MULT $\leq 8)$ and 15 literate persons (MULT $>8$ ). Participants aged 40-75 y, 21 were female. The pictograms obtained highest ANSI score for each direction (fig. 2) were chosen. Participants additionally suggested that a clock indicating time should be included in pictogram, for example 8 am for morning, $12 \mathrm{pm}$ for noon, $6 \mathrm{pm}$ for evening and $9 \mathrm{pm}$ for bedtime. The final pictogram was drawn as shown in fig. 3. Pictograms were printed a blue print on white paper, which could then be inserted into the standard zipped plastic packages routinely used in hospitals. The pictogram was divided into three parts. The first part of pictograms showed patients' name, date, medication name and indication, using standard text for Thai medical label. The second part indicated the time for taking medication during the day. Pharmacists had to circle the correct time of administration and shaded or painted a tablet picture based on the amount of tablets for each dose. The third part illustrated time in relation to meals whether to be taken before or after a meal. The information to which did not apply to the patient would be strikethrough by putting a cross with thick straight line.

Ultimately, 134 patients participated, composed of two groups with 67 participants each. Table 1 shows demographic data and clinical outcomes comparing the control and experimental group at day 0 . There was no difference noted between two groups at baseline. Overall, the average age of participants was $62.03 \pm$ 7.92 y. About $90 \%$ of them completed primary school. All of them had underlying disease(s). The average MULT score was $6.50 \pm 1.30$; therefore, they were classified as low literate patients.

At baseline, there was no statistical difference between the two groups in MULT score. The average sum score of MULT were $6.67 \pm 1.28$ and $6.30 \pm 1.40$, respectively. The score was less than 8 indicating there

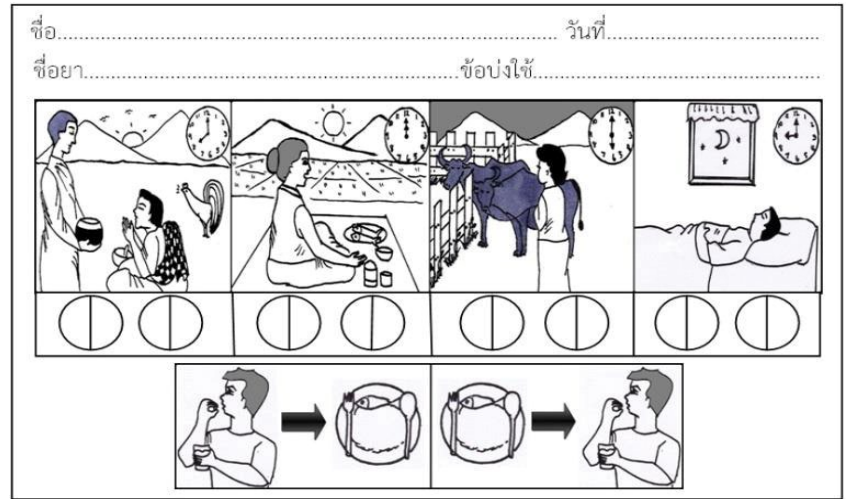

Fig. 3: The final pictogram

are low literate skills patients. There was no statistical difference between groups in each item.

At baseline, there was no statistical difference regarding the understanding of prescription directions score between the two groups. The average score of the control and experimental group at day 0 was $91.60 \pm 10.14$ and $89.40 \pm 14.27$, respectively. At the end of study, the average scores in both groups increased significantly $(p<0.001)$. However, the post test score of experimental group achieved a significantly increase over the control group ( $100 \pm 0.00$ vs. $98.10 \pm 3.64$; Table 2).

Patient adherence was assessed by the pill count method. It represented the percent of prescribed doses that the patient took during the interval of observation. At baseline, there was no statistical difference in patient adherence between two groups. As shown in Table 2, both groups showed statistically significant improvement at the end of study; however, the amount of patient adherence change in experimental group was increased statistically than control group.

The patient satisfaction scores were calculated and reported in fig. 4. The average score of patient satisfaction in each question was equal or greater than 4. Only question 1 that the average score was $3.63 \pm 1.14$, since participants felt the font size was not different from that on traditional label.

This is a well-designed study to construct the new pictograms used for Thai illiterates. The pictogram was designed by adapting from previous developed by USP-DI ${ }^{[15]}$ to northeastern culture relevant symbols. In the development of pictograms, phase I, exhibited that participants preferred symbols being relevant or close to northeastern Thai culture. This finding was consisted with a previous study. Dowse and Ehlers (2001) ${ }^{[18]}$ found that Africans had higher comprehension 
TABLE 1: DEMOGRAPHIC AND CLINICAL OUTCOMES AT BASELINE $(n=134)$

\begin{tabular}{|c|c|c|c|}
\hline Characteristics & Control $n=67$ & Experimental $n=67$ & p-value \\
\hline \multicolumn{4}{|l|}{ Gender } \\
\hline Female & $47(70.10)$ & $54(80.60)$ & $0.229^{a}$ \\
\hline Age in year, mean $\pm S D$ & $61.63 \pm 9.92$ & $62.99 \pm 6.40$ & $0.125^{\mathrm{b}}$ \\
\hline \multicolumn{4}{|l|}{ Occupation, n(\%) } \\
\hline Farmer & $40(59.70)$ & $28(41.80)$ & \multirow{4}{*}{$0.179^{a}$} \\
\hline Merchant & $1(1.50)$ & $1(1.50)$ & \\
\hline Housekeeper & $23(34.30)$ & $31(46.30)$ & \\
\hline Other & $3(4.50)$ & $7(10.40)$ & \\
\hline \multicolumn{4}{|l|}{ Education level, $n(\%)$} \\
\hline Primary school & $63(94.00)$ & $62(92.50)$ & \multirow{2}{*}{$1.000^{\mathrm{a}}$} \\
\hline Lower than primary school & $4(6.00)$ & $5(7.50)$ & \\
\hline \multicolumn{4}{|l|}{ number of medication prescribed } \\
\hline 1 & $4(6.00)$ & $2(3.00)$ & \multirow{5}{*}{$0.686^{\mathrm{a}}$} \\
\hline 2 & $15(22.40)$ & $17(25.40)$ & \\
\hline 3 & $21(31.30)$ & $16(23.90)$ & \\
\hline 4 & $14(20.90)$ & $14(20.90)$ & \\
\hline 5 & $13(19.40)$ & $18(26.90)$ & \\
\hline \multicolumn{4}{|l|}{ Diagnosis } \\
\hline Diabetes mellitus & 9 & 12 & $0.581^{\mathrm{a}}$ \\
\hline Hypertension & 11 & 14 & $0.480^{\mathrm{a}}$ \\
\hline Chronic kidney disease & 7 & 7 & $1.000^{\mathrm{a}}$ \\
\hline Cardiovascular disease & 5 & 3 & $0.592^{\mathrm{a}}$ \\
\hline Diabetes mellitus+hypertension & 13 & 10 & $0.612^{\mathrm{a}}$ \\
\hline $\begin{array}{l}\text { Diabetes mellitus+hypertension+chronic } \\
\text { kidney } \\
\text { disease }\end{array}$ & 22 & 21 & $0.887^{\mathrm{a}}$ \\
\hline Average MULT score, mean \pm SD & $6.67 \pm 1.28$ & $6.30 \pm 1.40$ & $0.681^{\mathrm{b}}$ \\
\hline
\end{tabular}

Note: 'a' analyzed by $\chi^{2}$-test, ' $\mathrm{b}$ ' analyzed by independent $t$-test

TABLE 2: THE AVERAGE UNDERSTANDING OF PRESCRIPTION DIRECTIONS SCORE AND SCORE OF PATIENT ADHERENCE

\begin{tabular}{lcccc}
\hline Groups & \multicolumn{2}{c}{ Average understanding of prescription directions score } & $\begin{array}{c}\text { Actual } \\
\text { change }\end{array}$ & P-value \\
\cline { 2 - 4 } & Pretest $($ mean $\pm S D)$ & Posttest (mean $\pm S D)$ & +5.50 & $<0.001^{\mathrm{a}}$ \\
\hline Control - traditional label $(\mathrm{n}=67)$ & $91.60 \pm 10.14$ & $100 \pm 0.00$ & +10.6 & $<0.001^{\mathrm{a}}$ \\
Experimental - pictogram label $(\mathrm{n}=67)$ & $89.40 \pm 14.27$ & $0.046^{\mathrm{b}}$ & & \\
p-value & $0.304^{\mathrm{b}}$ & & & \\
Score of patient adherence & & $95.84 \pm 4.72$ & +6.88 & $<0.001^{\mathrm{a}}$ \\
Control - traditional label $(\mathrm{n}=67)$ & $88.96 \pm 12.09$ & $97.41 \pm 3.62$ & +11.06 & $<0.001^{\mathrm{a}}$ \\
Experimental - pictogram label $(\mathrm{n}=67)$ & $86.35 \pm 12.56$ & $0.033^{\mathrm{b}}$ & & \\
$p$-value & $0.223^{\mathrm{b}}$ & & &
\end{tabular}

Note: 'a' analyzed by paired t-test, 'b' analyzed by independent t-test

of the locally developed pictures in comparison to the standardized pictograms. The participants in our finding suggested that local pictogram should present a clock, which would be useful for representing the time of taking medication and indicates the time at 8 am for morning, $12 \mathrm{pm}$ for noon, $6 \mathrm{pm}$ for evening and $9 \mathrm{pm}$ for bedtime. The low literate participants were able to clearly understand a clock face and those local pictogram on which clock faces appeared were generally well interpreted. The previous study found that a high proportion in South Africa regardless of their literacy level were able to tell the time from clock face. Conversely, Morrow et al. ${ }^{[25]}$ study reported the clock icons on local pictogram was complex to enhance understanding and could not overcome the advantage provided by the familiarity of the text-based format. The fourth phase was a RCT conducted in four provinces in the northeast, Thailand. At the beginning 


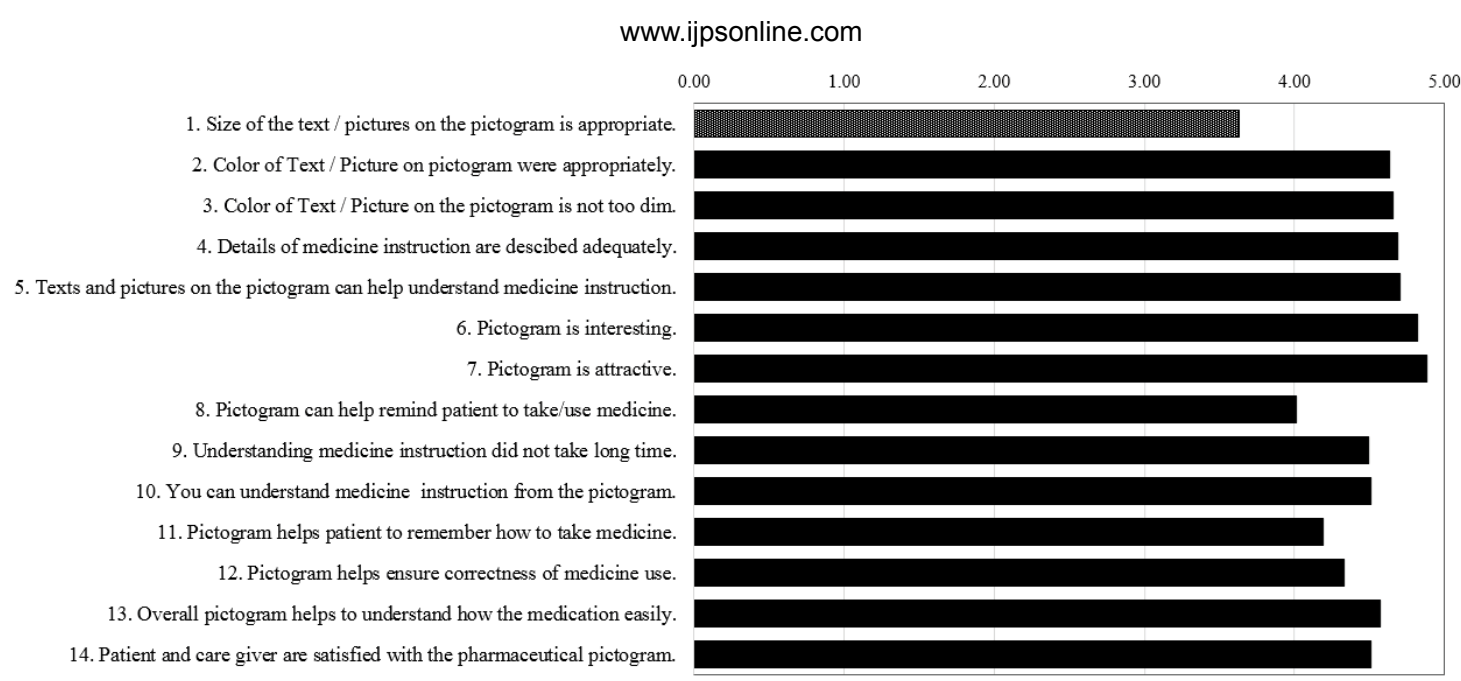

Fig. 4: Patient satisfaction with pictogram

$\mathrm{n}=67$

of the study, 136 low-literacy patients agreed to take part but 2 were dropped out. Therefore, 134 participants were retained to the completion of this study (fig. 1). The majority of patients were low income (75.37\%), correlating to previous studied, which reported higher prevalence of low-literacy found in low income groups ${ }^{[26]}$. Most of the participants completed the compulsory level of education, which was grade four. Regarding the understanding of prescription directions score, interventions used in both groups, appeared effective in achieving the goal of increase understanding of prescription directions. However, the change score in experimental group significantly increased over than control group (Table 2). A previous study done in Thailand reported that pictogram intervention produced significantly improved understanding of prescriptions ${ }^{[21]}$. Our findings confirmed the previous study, which showed pictograms help comprehension of instruction on prescriptions ${ }^{[2,27,28]}$. The results found that the participant pictogram group had understanding score higher than traditional labeling. The problem in patients with low literacy is their inability to read the text; therefore, in this population the pictograms should always be used with verbal counseling. The pharmacists were required to spend extra time to explain the pictogram labels. If patients such as these were not intensively counseled when they received their medications, and had not some means of recalling the instructions after leaving a hospital, appropriate medication-taking behavior would not have happened ${ }^{[21]}$.

Despite patient adherence, a traditional label and pictogram-based intervention resulted in improved adherence with the pictogram-based intervention significantly. Our finding was consistent with other studies regarding the use of pictogram instruction improving adherence among multiethnic, low socioeconomic status care givers ${ }^{[29]}$. Although, Chaijinda study ${ }^{[21]}$ reported that pictograms did not help improve patient comprehension of medication use; however, this study shows benefit of its use.

The satisfaction was evaluated in experimental group. The participants like images on the label and were satisfied with the color and the details of how drugs should be taken. Moreover, the pictogram can help them remember the method to take medication, remind them of the time to take medication correctly. Overall, the patients were satisfied with the labels and images that were on the labels. The images helped them to understand how to take the drug. Samerkum (2003) ${ }^{[30]}$ studied the effect of how the pictogram increased recognition of patient information to drug use. That causes the label to add a picture to remember the information of patients taking the drug because images are eye-catching and attract the attention of the patient rather than the words, especially if the patient has never seen the picture on the label. Chaijinda ${ }^{[21]}$ studied the development and evaluation of pictograms for low literacy patients in the northern part of Thailand and found that the participant prefer pictogram more than text labeling.

Limitation, participants in phase I-III were recruited based on convenience, which may cause selection bias to this study. Additionally, developing this pictogram involved with a small group of participants, thus may limit generalizability to apply in wider population. Although, health care professionals joined in early phases (I-II), but their expertise would have minimal 
impact on pictogram use since the effectiveness was proved by general public or patients in the latter phases (III-IV). The improvement of medication adherence and understanding of medicine use was minor, probably because participants already experienced in taking medications. Nonetheless, the greater impact could be assumed when applied to use with patients who are new medicine users. During measuring adherence, it was impossible to check if participants had discarded any pills before the follow-up. The random sequence number used in RCT was generated manually (drawing lots) but participants of both groups were similar. Therefore, effect of bias could be minimal.

In conclusion, objects or symbols used in designing pictograms should be simple, clear, culturally acceptable, and reflecting local tradition and habits. The local pictogram designed from this study was well-matched to patients in northeastern Thailand with low literacy. Results from RCT confirmed the benefit that the final pictogram significantly increased understanding of direction to use medications and medication adherence. Moreover, the final pictogram obtained high level of satisfaction from users. The findings suggest that the pictogram can potentially improve quality use of medicine in patients in northeastern Thailand with low literacy.

\section{Conflict of interest:}

Authors reported that there is no conflict of interest.

\section{Financial assistance and scholarship:}

Nil.

\section{REFERENCES}

1. Andrus MR, Roth MT. Health literacy: A review. Pharmacotherapy 2002;22:282-302.

2. Ngoh LN, Shepherd MD. Design, development, and evaluation of visual aids for communicating prescription drug instructions to nonliterate patients in rural Cameroon. Patient Educ Couns 1997;30:245-61.

3. Literacy Statistics trends 1985-2015 [cited 2016 Feb 5]. Available from: http:/www.uis.unesco.org/Education/ Documents/literacy-statistics-trends-1985-2015.pdf.

4. Angsuwattanakul B, Lerkiatbundit S. Development of Pictograms for Illiterate Patients Part 1: Instructions on How to Take Tablets. Thai J Pharm Pract 2014;6:42-60.

5. Health literacy and health behaviour. Available from: http:// www.who.int/healthpromotion/conferences/7gchp/track2/en/.

6. Barros IM, Alcântara TS, Mesquita AR, Santos AC, Paixão FP, Lyra DP. The use of pictograms in the health care: a literature review. Res Social Adm Pharm 2014;10:704-19.

7. Dowse R, Ehlers MS. Pictograms in pharmacy. Int J Pharm Pract 1998;6(2):109-18.
8. Houts PS, Witmer JT, Egeth HE, Loscalzo MJ, Zabora JR. Using pictographs to enhance recall of spoken medical instruction II. Patient Educ Couns 2001;43(3):231-42.

9. Chan $\mathrm{AH}$, Chan KW. Effects of prospective-user factors and sign design features on guessability of pharmaceutical pictograms. Patient Educ Couns 2013;90:268-75.

10. Thompson CA. Health illiteracy interferes with care. Am J Health Sys Pharm 2001;58:1294-5.

11. Barros IMC, Alcântara TS, Mesquita AR, Bispo ML, Rocha $\mathrm{CE}$, Moreira VP, et al. Understanding of pictograms from the United States Pharmacopeia Dispensing Information (USP-DI) among elderly Brazilians. Patient Prefer Adherence 2014;29:1493-501.

12. Clayton M, Syed F, Rashid A, Fayyaz U. Improving illiterate patients understanding and adherence to discharge medications. BMJ Qual Improv Rep 2012;1(1):1-4.

13. Davis TC, Federman AD, Bass PF, Jackson RH, Middlebrooks M, Parker RM, et al. Improving patient understanding of prescription drug label instructions. J Gen Intern Med 2009;24:57-62.

14. Dowse R, Ehlers MS. The influence of education on the interpretation of pharmaceutical pictograms for communicating medicine instructions. Int J Pharm Pract 2003;11:11-8.

15. USP Pictograms. Available from: http://www.usp.org/ usp-healthcare-professionals/related-topics-resources/ usppictograms/download-pictograms.

16. Develop pictograms specific to a culture. Available from: https://www.fip.org/www/index.php?page=mp_inst_develop.

17. Mansoor LE, Dowse R. Effect of pictograms on readability of patient information materials. Ann Pharmacother 2003;37(78):1003-9.

18. Dowse R, Ehlers MS. The evaluation of pharmaceutical pictograms in a low-literate South African population. Patient Educ Couns 2001;45:87-99.

19. Houts PS, Doak CC, Doak LG. Loscalzo MJ. The role of pictures in improving health communication: a review of research on attention, comprehension, recall, and adherence. Patient Educ Couns 2006;61(2):173-90.

20. Kassam R, Vaillancourt CR, Collins JB. Pictographic instructions for medications: do different cultures interpret them accurately? Int J Pharm Pract 2004;12:199-209.

21. Chaijinda K, Nilaward K, Chuamanochan P, Awiphan R. Development and evaluation of pictorial labeling system for Northern Thai patients with low literate skills [dissertation]. Chiang Mai. Chiang Mai University; 2007.

22. American National Standard Institute. Accredited standard on safety colours, signs, symbols, labels, and tags. Vol. Z535. Washington (DC): National Electrical Manufacturers Association; 1991.

23. Joshi Y, Kothiyal P. A pilot study to evaluate pharmaceutical pictograms in a multispecialty hospital in Dehradun. J Young Pharm 2011;3:163-6.

24. Montagne M. Pharmaceutical pictograms: a model for development and testing for comprehension and utility. Res Social Adm Pharm 2013;9:609-20.

25. Morrow DG, Leirer VO, Andrassy JM. Using icons to convey medication schedule information. Appl Ergon 1996;27:267-75.

26. Gazmararian JA, Baker DW, Williams MV, Parker RM, Scott TL, Green DC, et al. Health literacy among Medicare enrollees in a managed care organization. JAMA 1999;281:545-51. 
27. Morrow DG, Hier CM, Menard WE, Leirer VO. Icons improve older and younger adults' comprehension of medication information. J Gerontol B Psychol Sci Soc Sci 1998;53:24054.

28. Dowse R, Ehlers M. Medicine labels incorporating pictograms: do they influence understanding and adherence? Patients Educ Couns 2005;58:63-70.

29. Yin HS, Dreyer BP, Schaick LV, Foltin GL, Dinglas C, Mendelsohn AL. Randomized Controlled Trial of a Pictogram-
Based Intervention to Reduce Liquid Medication Dosing Errors and Improve Adherence Among Caregivers of Young Children. Arch Pediatr Adolesc Med 2008;162:814-22.

30. Samerkum K, Kanman K. The development and evaluation of pictorial auxiliary labels in increasing recall of medication information: a case study among outpatients, Chiang Mai Neurological Hospital and Nakornping Hospital, Chiang Mai Province [dissertation]. Chiang Mai: Chiang Mai University; 2003. 